\title{
On finite time observer design for multicellular converter
}

\author{
Michael Defoort, Mohamed Djemaï, Thierry Floquet and Wilfrid Perruquetti
}

\begin{abstract}
The aim of this paper is to estimate the capacitor's voltages by tacking the hybrid behavior of the multicellular converter into account. A nonlinear finite time observer is given in order to solve this problem. The stability and properties of the proposed homogeneous finite time observer are studied using Lyapunov theory. Our approach enables the stabilization of the observation errors in spite of the presence of perturbations. Simulations highlight the efficiency of the proposed strategy.

Index Terms - multicellular converter, higher order sliding mode, nonlinear observer, switched system, finite time stability.
\end{abstract}

\section{INTRODUCTION}

Power electronics have been evolving through the last decades due to the developments of the semiconductor power components and new systems of energy conversion [6]. Among these systems, multicellular converters, which appeared at the beginning of the 90's [17], are based on the association in series of the elementary cells of commutation. During this last decade, these systems become more and more attractive to industrial applications, especially in highpower applications [18]. Indeed, the harmonic contents of the waveforms are improved compared to the classical two levels converter technology using the same switching frequency. Furthermore, this structure enables the reduction of the losses due to commutations of power semiconductors while allowing cheap electronics components of large diffusion.

To benefit as well as possible from the large potential of the multicellular structure, an appropriate distribution of the voltages crossing each cell is needed. These voltages are generated when a suitable control of switches is applied in order to obtain a specific value. The control of switches allows to cancel the harmonics at the switching frequency and to reduce the ripple of the chopped voltage [9]. Anyway, the knowledge of the capacitor voltages is always needed. Furthermore, it is important to note that the use of physical extra sensors in order to measure such voltages increases the cost and the complexity of the system. That is why, its estimation by means of an observer becomes an attractive and economical solution.

Different observer based methods have been developed for nonlinear systems such as, for instance, adaptive observers [9], observers by input-output injection [10], higher order sliding mode observers [15], [8], [5], [21], [7] or finite time observers [19], [20].

M. Defoort and M. Djemaï (e-mail: \{michael.defoort, mohamed.djemai\}@univ-valenciennes.fr) are with Univ Lille Nord de France, F-59000 Lille, CNRS, FRE 3304, F-59313 Valenciennes, UVHC, LAMIH, F-59313 Valenciennes, France

T. Floquet and W. Perruquetti are with LAGIS, Ecole Centrale de Lille, F-59651 Villeneuve d'Ascq and Equipe Projet ALIEN, INRIA, France
For the first order sliding modes, it is common to deal with the issues of stability, robustness and convergence rate of the equilibrium by means of a Lyapunov approach [23]. For higher sliding mode and finite time observers, the proof of stability is usually done using geometric homogeneity based methods. Hence, the estimation of the convergence time and the study of robustness properties looks problematic. In [4], a strong Lyapunov function for the super-twisting algorithm is proposed, allowing the study of stability, robustness and convergence rate of the equilibrium.

In this paper, a Lyapunov function will be designed, based on a quadratic like function, in order to deeply study the reaching time estimation and robustness of the homogenous finite time observer proposed in [20]. This observer is then applied in order to estimate the capacitor voltages in a multicellular converter whose hybrid behavior, due to switches, increases the difficulty. The results are illustrated with some simulations.

\section{Multicellular COnVerter MOdeling}

The multicellular converter is based on the combination of elementary cells of commutation. Figure 1 depicts the topology of a converter with $p$ independent commutation cells. The independency is due to the $(p-1)$ internal capacitors and can be considered only for a few switching periods. As a matter of fact, the current flows from the source $E$ to the output $I$ through different converter switches. The multicellular converter shows, by its structure, a hybrid behavior due to discrete variables (i.e. switching or commutation logic) [1]. Note that because of the presence of capacitors, there are also continuous variables (i.e. currents and voltages).

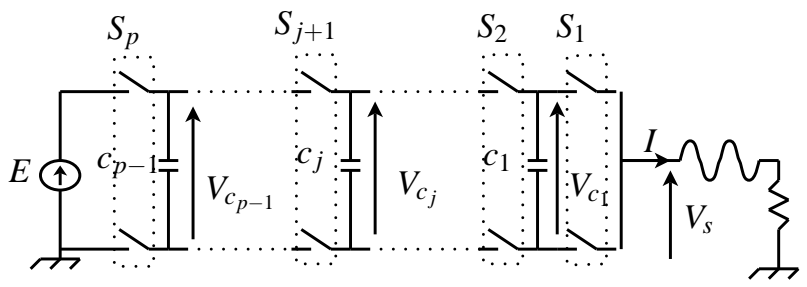

Fig. 1. Multicellular converter

It is important to highlight that the electrical switches constraints should be similar. This requirement implies a unique voltage switch constraint of $\frac{E}{p}$. Thus, it is necessary to ensure an equilibrated distribution of the capacitor voltages. Under these conditions, the reference voltage of the capacitor $j(j=\{1, \ldots, p-1\})$ is:

$$
V_{c_{j}, r e f}=j \frac{E}{p}
$$


The dynamics of the converter, with a load consisting in a resistance $R$ and an inductance $L$, is given by the following differential equations:

$$
\left\{\begin{array}{c}
\dot{I}=-\frac{R}{L} I+\frac{E}{L} S_{p}-\sum_{j=1}^{p-1} \frac{V_{c_{j}}}{L}\left(S_{j+1}-S_{j}\right) \\
\dot{V}_{c_{j}}=\frac{I}{c_{j}}\left(S_{j+1}-S_{j}\right), \quad j=1, \ldots, p-1
\end{array}\right.
$$

where $I$ is the load current, $c_{j}$ is the capacitance, $V_{c_{j}}$ is the voltage in the $j$-th capacitor and $E$ is the voltage of the source. Each commutation cell is controlled by the binary input signal $S_{j} \in\{0,1\}$. Signal $S_{j}=1$ means that the upper switch of the $j$-th cell is "on" and the lower switch is "off" whereas $S_{j}=0$ means that the upper switch is "off" and the lower switch is "on".

The discrete control input can be described as follows:

$$
\left\{\begin{array}{l}
u_{j}=S_{j+1}-S_{j}, \quad j=1 \ldots p-1 \\
u_{p}=S_{p}
\end{array}\right.
$$

Assuming that only the load current $I$ can be measured (i.e. $y=I$ ), the system (1) can be represented as follows:

$$
\left\{\begin{aligned}
\dot{I} & =-\frac{R}{L} I+\frac{E}{L} u_{p}-\sum_{j=1}^{p-1} \frac{V_{c_{j}}}{L} u_{j} \\
\dot{V}_{c_{j}} & =\frac{I}{c_{j}} u_{j}, \quad j=1, \ldots, p-1 \\
y & =I
\end{aligned}\right.
$$

Using the hybrid system formalism [16], one can describe the system (3) as:

$$
\left\{\begin{array}{l}
\dot{x}=f(x, u) \\
y=h(x, u)
\end{array}\right.
$$

where $x=\left[I, V_{c_{1}}, \ldots, V_{c_{p-1}}\right]^{T} \in \mathbb{R}^{p}$ is the continuous state, $u=\left[u_{1}, \ldots, u_{p}\right]^{T}$ is the applied control input which only takes discrete values. The functions $f(x, u)=A(u) x+B(u)$ and $h(x, u)=C x$ are vector fields defined as:

$$
\begin{gathered}
A(u)=\left[\begin{array}{cccc}
-\frac{R}{L} & -\frac{u_{1}}{L} & \ldots & -\frac{u_{p-1}}{L} \\
\frac{u_{1}}{c_{1}} & 0 & \ldots & 0 \\
\vdots & \vdots & \ddots & \vdots \\
\frac{u_{p-1}}{c_{p-1}} & 0 & \ldots & 0
\end{array}\right] \\
B(u)=\left[\frac{E}{L} u_{p}, 0, \ldots, 0\right]^{T} \\
C=[1,0, \ldots, 0]
\end{gathered}
$$

\section{OBSERVABILITY ANALYSIS}

This section is devoted to the observability analysis of the capacitor voltages $V_{c_{j}}(j=\{1, \ldots, p-1\})$ from the measurement of the current $I$ and the knowledge of the control input sequence $u$. This analysis can be done using the two following approaches [2].

\section{A. Approach 1: Nonlinear observation theory}

In this approach, the converter is considered as a nonlinear continuous system and tools from nonlinear observation theory [11] are used to study the observability.

Considering the system (3), one can see that there are several operating switching modes for which the state $x$ is not observable. For instance, if $u_{1}=u_{2}=\cdots=u_{p-1}=0$, the voltages $V_{c_{j}}(\forall j=\{1, \ldots, p-1\})$ become completely unobservable. Nevertheless, these operating switching modes occur only during some part of the control sequence. Indeed, if it occurs for all the control sequences then the physical interest is low because the multicellular converter does not operate.

From the observability rank condition [13] of the system (3), it follows that at most two components of $x$ are simultaneously observable. System (3) can be written as follows:

$$
\left\{\begin{array}{l}
\dot{I}=-\frac{R}{L} I+\frac{E}{L} u_{p}+V_{s} \\
\dot{V}_{s}=-\sum_{j=1}^{p-1} \frac{I}{L c_{j}} u_{j}^{2}
\end{array}\right.
$$

with $V_{s}=-\frac{1}{L} \sum_{j=1}^{p-1} u_{j} V_{c_{j}}$. From the observability rank condition of system (5), one can deduce that $I$ and $V_{s}$ can be simultaneously observable.

From the estimation of $V_{s}$, one can deduce the capacitor voltages $V_{c_{1}}, V_{c_{2}}, \ldots, V_{c_{p}-1}$. Indeed, since $V_{c_{j}}(j=\{1, \ldots, p-$ 1\}) slowly varies during the measurement time interval, one can obtain $p-1$ measurements of $V_{s}$ (i.e. $V_{s}^{1}, V_{s}^{2}, \ldots V_{s}^{p-1}$ ) such that:

$$
\left[\begin{array}{c}
V_{s}^{1} \\
\vdots \\
V_{s}^{p-1}
\end{array}\right]=-\frac{1}{L}\left[\begin{array}{ccc}
u_{1}^{1} & \ldots & u_{p-1}^{1} \\
\vdots & \ddots & \vdots \\
u_{1}^{p-1} & \ldots & u_{p-1}^{p-1}
\end{array}\right]\left[\begin{array}{c}
V_{c_{1}} \\
\vdots \\
V_{c_{p-1}}
\end{array}\right]
$$

is invertible.

\section{B. Approach 2: Z-observability theory}

Here, the observability properties of the state vector is studied from the concept of $Z$-observability for switched systems [14]. This approach uses the fact that the multicellular converter belongs to a particular class of switching hybrid systems. For such a class, the observation concept is linked to the switches. Hence, it is important to give the following definitions.

Definition 1: [16] A hybrid time trajectory is a finite sequence $T_{n}=\left\{I_{i}\right\}_{i=0, N}$ such that:

- $I_{i}=\left[t_{i, 0}, t_{i, 1}\right)$, for all $0 \leq i \leq N$

- For all $0 \leq i \leq N, t_{i, 1}=t_{i+1,0}$

- $t_{0,0}=t_{\text {ini }}$ and $t_{N, 1}=t_{\text {end }}$

Furthermore, $\left\langle T_{N}\right\rangle$ is defined as the ordered list of $u$ associated to $T_{N}$, i.e. $\left\{u^{i}\right\}_{i=0, N}$ where $u^{i}$ is the value of $u$ on the interval $I_{i}$.

Definition 2: [14] For system (4), the function $z=Z(t, x)$ is said to be $Z$-observable with respect to the hybrid time 
trajectory $T_{N}$ and $\left\langle T_{N}\right\rangle$, if for all trajectories $(t, x, u)$ and $\left(t, x^{\prime}, u^{\prime}\right)$ defined in $\left[t_{\text {ini }}, t_{\text {end }}\right]$, the equality $h(x, u)=h\left(x^{\prime}, u^{\prime}\right)$ implies $Z(t, x)=Z\left(t, x^{\prime}\right)$.

Theorem 1: [14] Consider system (4) and a fixed hybrid time trajectory $T_{N}$ and $\left\langle T_{N}\right\rangle$. Suppose that $z=Z(t, x)$ is always continuous under any admissible control input. If there is a sequence of linear projections $\left\{P_{i}\right\}_{i=0, N}$ such that:

- for all $0 \leq i \leq N, P_{i} Z(t, x)$ is $Z$-observable for $t \in I_{i}$,

- $\operatorname{Rank}\left(\left[P_{0}^{T}, \ldots, P_{N}^{T}\right]\right)=\operatorname{dim}(z)$,

- $\frac{d \bar{P}_{i} Z(t, x)}{d t}=0$ for $t \in I_{i}$ where $\left\{\bar{P}_{i}^{T}, P_{i}^{T}\right\}$ has a full rank

Then, $z$ is $Z$-observable with respect to the hybrid time trajectory $T_{N}$ and $\left\langle T_{N}\right\rangle$.

Remark 1: The first condition of Theorem 1 implies that there is at least a time interval in which the variable $P_{i} Z(t, x)$ is $Z$-observable; whereas the second one implies that all the components of $Z$ are observable in a given time interval of the hybrid time trajectory $T_{N}$. The third condition requires that components of $Z$ which are not observable in $I_{i}$ must remain constant within this time interval. It prevents from the loose of observability and from the "re-observation" of variables which have been already estimated.

The application of Theorem 1 to the multicellular converter yields the following result:

Lemma 1: Consider the system (3) and the function $z=$ $x$. Then, $z$ is $Z$-observable with respect to the hybrid time trajectory $T_{N}$ and $\left\langle T_{N}\right\rangle=\left\{u^{i}\right\}_{i=0, N}$, if the vectors $\left\{u^{i}\right\}_{i=0, N}$ generate the space $\mathbb{R}^{p-1}$, where

$$
u^{i}=u
$$

on the time interval $I_{i}$.

Remark 2: Lemma 1 ensures that the current crosses through all the capacitors; roughly speaking, in a linear independent form. In other words, after $p-1$ time intervals (i.e. $p-1$ control sequences) the measurement of the load current $I$ allows to obtain a set of $(p-1)$ linearly independent equations with respect to the voltages in the $(p-1)$ capacitors; which enables to estimate the capacitors of the converter. This result is, in essence, similar as the result presented in Approach 1. Indeed, it is needed at least $p-1$ time intervals whose corresponding sequences $u^{i}$ are linearly independent, in order to estimate $V_{c_{j}}, \forall j \in\{1, \ldots, p-1\}$.

\section{CApacitor Voltages estimation}

Hereafter, a finite time observer is designed in order to estimate the capacitor voltages of the multicellular converter by using only the measurement of the current $I$. Due to the particular structure of the observer, finite time stability of the observation error is shown using Lyapunov stability arguments.

\section{A. Finite time observer design}

Assuming that the current $I$ is measured, the finite time observer for the system (5) is designed as follows:

$$
\left\{\begin{array}{l}
\dot{\hat{I}}=-\frac{R}{L} I+\frac{E}{L} u_{p}+\hat{V}_{s}+k_{1} \sum_{j=1}^{p-1}\left|u_{j}\right|\lceil I-\hat{I}\rfloor^{\alpha} \\
\dot{\hat{V}}_{s}=-\sum_{j=1}^{p-1} \frac{I}{L c_{j}} u_{j}^{2}+K_{2}\lceil I-\hat{I}\rfloor^{2 \alpha-1}
\end{array}\right.
$$

The positive constants $k_{1}, K_{2}$ and $\alpha$ will be defined hereafter. The continuous function $\lceil a\rfloor^{b}$ is the function described as:

$$
\forall a \in \mathbb{R}, \forall b \geq 0, \quad\lceil a\rfloor^{b}=|a|^{b} \operatorname{sign}(a)
$$

Define the observation errors as:

$$
\left\{\begin{array}{l}
e_{1}=I-\hat{I} \\
e_{2}=V_{s}-\hat{V}_{s}
\end{array}\right.
$$

Equations (5)-(7) yield the dynamics of the observation error as:

$$
\left\{\begin{array}{l}
\dot{e}_{1}=e_{2}-k_{1} \sum_{j=1}^{p-1}\left|u_{j}\right|\left\lceil e_{1}\right\rfloor^{\alpha} \\
\dot{e}_{2}=-K_{2}\left\lceil e_{1}\right\rfloor^{2 \alpha-1}
\end{array}\right.
$$

In order to simplify the system (10), one can note:

$$
K_{1}=k_{1} \sum_{j=1}^{p-1}\left|u_{j}\right|
$$

Hence, the dynamics (10) can be written as follows:

$$
\left\{\begin{array}{l}
\dot{e}_{1}=e_{2}-K_{1}\left\lceil e_{1}\right\rfloor^{\alpha} \\
\dot{e}_{2}=-K_{2}\left\lceil e_{1}\right\rfloor^{2 \alpha-1}
\end{array}\right.
$$

Through this paper, it is assumed that:

- there is $T_{N}$ such that $z=x$ is $Z$-observable with respect to the hybrid time trajectory of system (1).

- there is a constant $\tau>0$ such that for any time interval $I_{i},\left|t_{i, 1}-t_{i, 0}\right|$ is larger than $\tau$.

Remark 3: Since $u$ remains constant on the time interval $I_{i}$, one should guarantee that the reaching time $t_{i, 0}<T_{i}<t_{i, 1}$ onto the manifold $\left\{e=\left[e_{1}, e_{2}\right]^{T} \mid e_{1}=0, e_{2}=0\right\}$ is lower than $\tau$. According to the above assumptions, there is a hybrid time trajectory such that the capacitor voltages are observable. Then, since $e_{2}$ equals to zero on the time interval $\left[T_{i}, t_{i, 1}\right]$, by the means of the procedure defined in Section III, one can obtain an estimation of the capacitor voltages, i.e. $V_{c_{j}}, \forall j=\{1, \ldots, p-1\}$.

According to Remark 3, the objective, i.e. the estimation of the capacitor voltages, is achieved when the finite time stabilization of the system (12) is guaranteed.

Consider some perturbations $\widetilde{f}(e)=\left[\widetilde{f}_{1}\left(e_{1}\right), \widetilde{f}_{2}\left(e_{1}\right)\right]^{T} \in$ $\mathbb{R}^{2}$ on the system (3) due to resistance uncertainties for instance. In this case, the dynamics of the observation errors (12) is replaced by:

$$
\left\{\begin{array}{l}
\dot{e}_{1}=e_{2}-K_{1}\left\lceil e_{1}\right\rfloor^{\alpha}+\widetilde{f}_{1}\left(e_{1}\right) \\
\dot{e}_{2}=-K_{2}\left\lceil e_{1}\right\rfloor^{2 \alpha-1}+\widetilde{f}_{2}\left(e_{1}\right)
\end{array}\right.
$$


Theorem 2: Suppose that the perturbations of the system (13) satisfy:

$$
\left\{\begin{array}{l}
\tilde{f}_{1}(0)=0 \\
\left|\tilde{\widetilde{f}}_{1}\left(e_{1}\right)\right| \leq \delta_{1}\left|e_{1}\right|^{2 \alpha-1} \\
\left|\widetilde{f}_{2}\left(e_{1}\right)\right| \leq \delta_{2}\left|e_{1}\right|^{2 \alpha-1}
\end{array}\right.
$$

for some constants $\delta_{1} \geq 0$ and $\delta_{2} \geq 0$.

Consider the matrix $A_{0}$ :

$$
A_{0}=\left[\begin{array}{cc}
-\alpha K_{1} & \alpha \\
-K_{2} & 0
\end{array}\right]
$$

where $\frac{1}{2} \leq \alpha<1$ and the gains $K_{1}>0, K_{2}>0$ are chosen such that matrix $A_{0}$ is Hurwitz and are sufficiently large. Then, the system (13) is globally finite-time stable.

Proof: Consider the following vector:

$$
\xi=\left[\begin{array}{l}
\xi_{1} \\
\xi_{2}
\end{array}\right]=\left[\begin{array}{c}
\left\lceil e_{1}\right\rfloor^{\alpha} \\
e_{2}+\widetilde{f}_{1}
\end{array}\right]
$$

Define the candidate Lyapunov function as:

$$
V(\xi)=\xi^{T} P \xi
$$

$P=P^{T}=\left[\begin{array}{ll}p_{1} & p_{3} \\ p_{3} & p_{2}\end{array}\right]>0$ is the solution of the following algebraic Lyapunov equation:

$$
A_{0}^{T} P+P A_{0}=-Q
$$

where $Q=Q^{T}>0$ is a positive definite matrix.

One can note that $V(\xi)$ is continuous, positive definite and radially unbounded in $\mathbb{R}^{2}$. Furthermore, it is differentiable everywhere except on the manifold $\left\{e_{1}=0\right\}$. Since the trajectory of system (12) cannot stay on this set, before reaching the origin, the derivative of $V$ can be calculated as in the conventional way (see [12] for further details).

Since

$$
\begin{aligned}
\dot{\xi} & =\left[\begin{array}{c}
\alpha\left|e_{1}\right|^{\alpha-1}\left(e_{2}-K_{1}\left\lceil e_{1}\right\rfloor^{\alpha}+\widetilde{f}_{1}\right) \\
-K_{2}\left\lceil e_{1}\right\rfloor^{2 \alpha-1}+\dot{\widetilde{f}}_{1}+\widetilde{f}_{2}
\end{array}\right] \\
& =\left|e_{1}\right|^{\alpha-1}\left[\begin{array}{c}
\alpha\left(\xi_{2}-K_{1} \xi_{1}\right) \\
-K_{2} \xi_{1}
\end{array}\right]+\left[\begin{array}{c}
0 \\
\dot{\widetilde{f}}_{1}+\widetilde{f}_{2}
\end{array}\right] \\
& =\left|e_{1}\right|^{\alpha-1} A_{0} \xi+\left[\begin{array}{c}
0 \\
\dot{\widetilde{f}}_{1}+\widetilde{f}_{2}
\end{array}\right]
\end{aligned}
$$

the time derivative of $V$ along the solutions of system (13) is given by:

$$
\dot{V}=-\frac{1}{\left|e_{1}\right|^{1-\alpha}}\left(\xi^{T} Q \xi+2 \xi^{T} P\left[\left|e_{1}\right|^{1-\alpha}\left(\dot{\widetilde{f}}_{1}+\widetilde{f}_{2}\right)\right]\right)
$$

where

$$
2 \xi^{T} P\left[\begin{array}{c}
0 \\
\dot{\widetilde{f}}_{1}+\widetilde{f}_{2}
\end{array}\right]=2\left(\dot{\widetilde{f}}_{1}+\widetilde{f}_{2}\right)\left(p_{3} \xi_{1}+p_{2} \xi_{2}\right)
$$

Using the bound on the perturbation (14), it can be shown that:

$$
2 \xi^{T} P\left[\begin{array}{c}
0 \\
\left|e_{1}\right|^{1-\alpha}\left(\dot{\widetilde{f}}_{1}+\widetilde{f}_{2}\right)
\end{array}\right] \leq 2\left(\delta_{1}+\delta_{2}\right)\left(p_{3} \xi_{1}^{2}+p_{2}\left|\xi_{1}\right|\left|\xi_{2}\right|\right)
$$

Since, for all $\varepsilon>0$,

$$
p_{2}\left|\xi_{1}\right|\left|\xi_{2}\right| \leq \frac{\varepsilon}{2} \xi_{1}^{2}+\frac{p_{2}^{2}}{2 \varepsilon} \xi_{2}^{2}
$$

One has:

$$
2 \xi^{T} P\left[\begin{array}{c}
0 \\
\left|e_{1}\right|^{1-\alpha}\left(\dot{\widetilde{f}}_{1}+\widetilde{f}_{2}\right)
\end{array}\right] \leq \mu_{1}^{2} \xi_{1}^{2}+\mu_{2}^{2} \xi_{2}^{2}
$$

with $\mu_{1}=\left(\delta_{1}+\delta_{2}\right)\left(2\left|p_{3}\right|+\varepsilon\right)$ and $\mu_{2}=\left(\delta_{1}+\delta_{2}\right) \frac{p_{2}^{2}}{\varepsilon}$. Thus,

$$
\begin{aligned}
\dot{V} & \leq-\frac{1}{\left|e_{1}\right|^{1-\alpha}} \xi^{T} \widetilde{Q} \xi \\
& \leq-\frac{1}{\left|e_{1}\right|^{1-\alpha}} \lambda_{\min }\{\widetilde{Q}\}\|\xi\|^{2}
\end{aligned}
$$

where

$$
\widetilde{Q}=Q+\left[\begin{array}{cc}
\mu_{1} & 0 \\
0 & \mu_{2}
\end{array}\right]
$$

Hence, if the gains $K_{1}$ and $K_{2}$ are chosen such that $\widetilde{Q}>0$, then $\dot{V}$ is negative definite.

From the standard inequality for quadratic forms, one can deduce that:

$$
\lambda_{\min }\{P\}\|\xi\|^{2} \leq V(\xi) \leq \lambda_{\max }\{P\}\|\xi\|^{2}
$$

where $\|\xi\|$ is the Euclidean norm.

Using (24) and $0<\frac{1-\alpha}{2 \alpha}$, one can obtain:

$$
\left|e_{1}\right|^{1-\alpha} \leq\|\xi\|^{\frac{1-\alpha}{\alpha}} \leq \frac{V^{\frac{1-\alpha}{2 \alpha}}(\xi)}{\lambda_{\min }^{\frac{1-\alpha}{2 \alpha}}\{P\}}
$$

As $\alpha>\frac{1}{2}$, one can deduce that $V(\xi)$ is a strong Lyapunov function for the system (13) since:

$$
\dot{V} \leq-\gamma(\widetilde{Q}) V^{\frac{3 \alpha-1}{2 \alpha}}
$$

with

$$
\gamma(\widetilde{Q})=\frac{\lambda_{\min }\{\widetilde{Q}\} \lambda_{\min }^{\frac{1-\alpha}{2 \alpha}}\{P\}}{\lambda_{\max }\{P\}}
$$

Since the transformation (15) is continuous, it follows that $\xi$ converges to zero in finite time, then, $e_{1}$ and $e_{2}$ converge to 0 and $-\widetilde{f}_{1}$, respectively.

Using the property of perturbation $\widetilde{f}_{1}$ given in (14), it follows that a trajectory of the system (13) starting from $e_{0} \in \mathbb{R}^{2}$ converges to the origin in finite time and reaches that point at most after:

$$
T=\frac{2 \alpha}{3 \alpha-1} \frac{V^{\frac{3 \alpha-1}{2 \alpha}}\left(e_{0}\right)}{\gamma(\widetilde{Q})}
$$

Remark 4: It is interesting to note that the limit case $\alpha=1$ corresponds to the Luenberger observer and allows an asymptotic convergence of the observation errors. The case $\alpha=\frac{1}{2}$ corresponds to the classical super-twisting observer. 


\section{Simulation Results}

In order to illustrate the performance of the proposed finite time observer, we consider here a 3 cells converter connected to an $R L$ load. This converter can be modeled by the system (1) where $p=3$. Its parameters are as follows: $c_{1}=c_{2}=$ $40.10^{-6} F, R=131 \Omega, L=0.001 H$.

The voltage of the source is $E=30 \mathrm{~V}$. The inputs of the switches $u_{j}, j=\{1,2,3\}$ are generated for the hybrid time trajectory given in Fig. 2. This sequence of control is periodic with a period of $0.2 \mathrm{~ms}$ and satisfies the assumptions of Lemma 1 . Hence, $z=x$ is $Z$-observable with respect to the hybrid time trajectory for system (1). Moreover, one can see in Fig. 4 that $V_{c_{1}}$ ( $V_{c_{2}}$ resp.) remains constant during a certain time interval. The chopping frequency and the sampled period are $F=5 \mathrm{kHz}$ and $T=5 \cdot 10^{-6} \mathrm{~s}$, respectively.

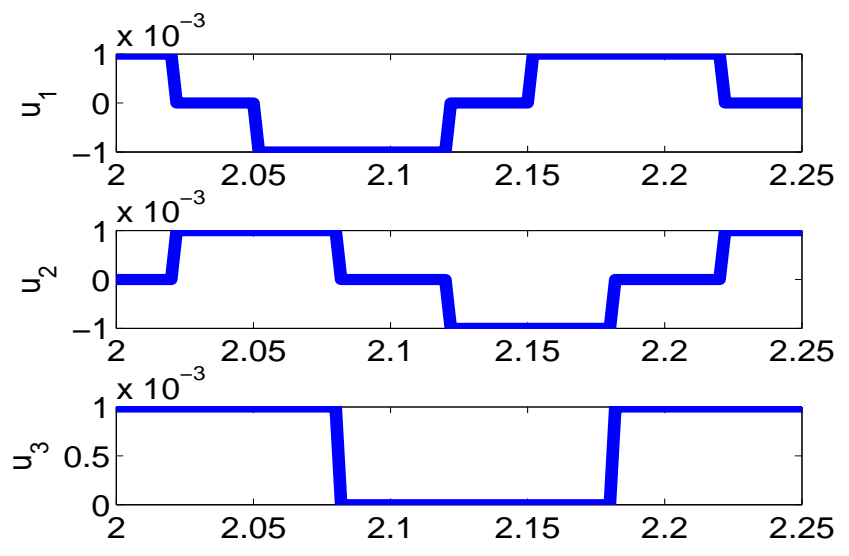

Fig. 2. Applied control inputs

The available measurement is the current $I$. The problem of the capacitor voltages estimation can be viewed as the finite-time stabilization of the system (12). Applying Theorem 2, one can set the parameters of the observers as follows: $K_{2}=1500$ and $K_{1}=500$.

Figures 3-5 depict the results obtained with the finite time observer when $\alpha=\frac{3}{4}$ is selected. One can note the high convergence rate of the observer errors. One can see the equal distribution of the voltage constraints on each cells (i.e. steady conditions $\frac{E}{3}=10 \mathrm{~V}$ for $V_{c_{1}}$ and $\frac{2 E}{3}=20 \mathrm{~V}$ for $V_{c_{2}}$ ). It is worth noticing that the high frequency oscillations are of low amplitude. It is interesting to note in Fig. 6 that the finite time observer gives good results in terms of robustness properties with respect to resistance variation $(25 \%$ around its nominal value).

\section{CONCLUSION}

In this paper, a nonlinear finite time observer has been designed for a multicellular converter. The problem of the capacitor voltages estimation is solved. A Lyapunov function has been introduced in order to study the stability and properties of the proposed homogeneous finite time observer.
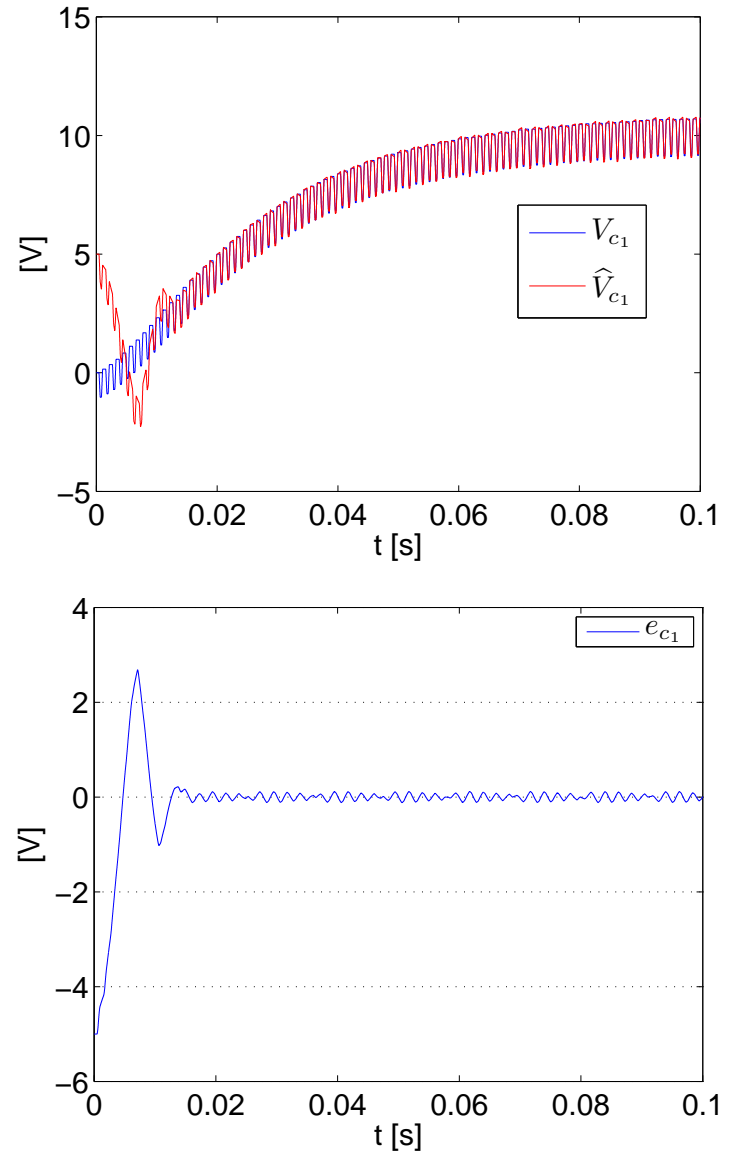

Fig. 3. Observation error in $V_{c_{1}}$ for the finite time observer using $\alpha=\frac{3}{4}$

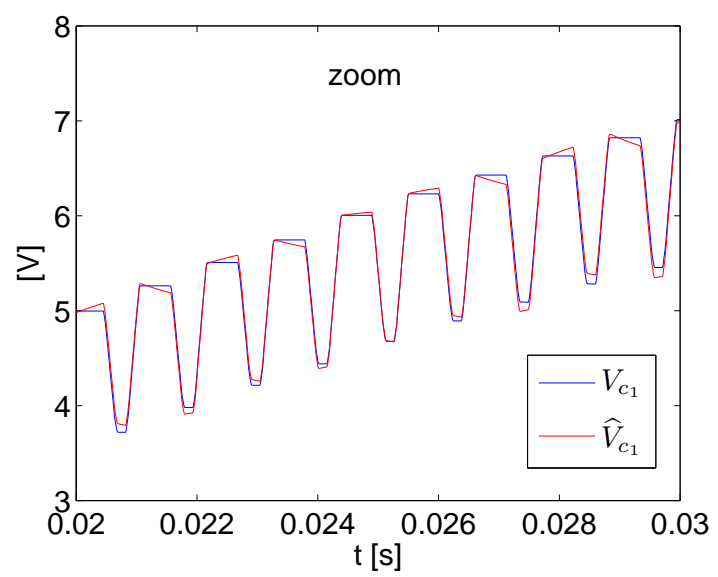

Fig. 4. Zoom

\section{REFERENCES}

[1] Barbot J.P., Saadaoui H., Djemaï M. and Manamanni N., "Nonlinear observer for autonomous switching systems with jumps", Nonlinear Analysis: Hybrid Systems pp. 537-547, 2007.

[2] Benmansour K., "Realisation d'un banc d'essai pour la Commande et l'Observation des Convertisseurs Multicellulaires Serie: Approche Hybride", PhD Thesis, Cergy Pontoise University, 2009.

[3] Bensaid R., Fadel M. and Meynard T., "Observer design for a three 

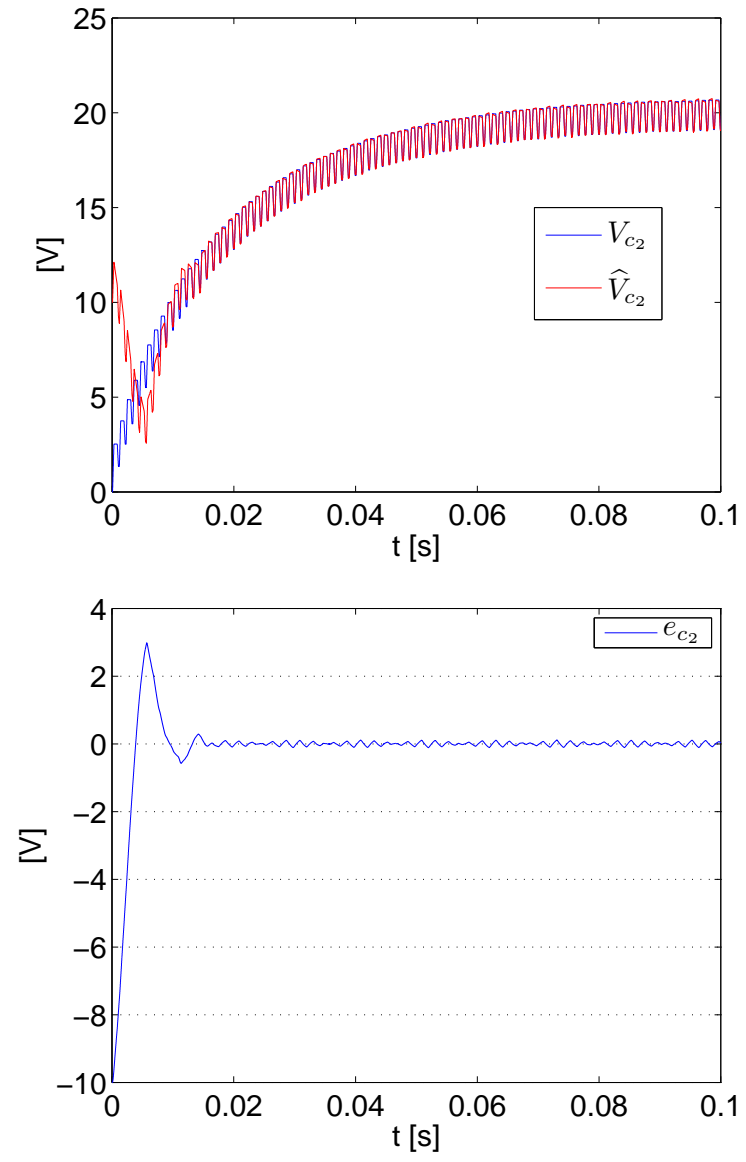

Fig. 5. Observation error in $V_{c_{2}}$ for the finite time observer using $\alpha=\frac{3}{4}$

cells chopper using discrete-time model". Electromotion, 2, pp. 689694, 1999.

[4] Davila M., Moreno J. and Fridman L., "Optimal Lyapunov Function Selection for Reaching Time Estimation of Super Twisting Algorithm", IEEE Conference on Decision and Control, 2009.

[5] Defoort M., Nollet F., Floquet T. and Perruquetti W., "A third order sliding mode controller for a stepper motor", IEEE Trans. on Industrial Electronics, 56(9), pp. 3337-3346 , 2009.

[6] Erickson R. and Maksimovic D., "Fundamentals of Power Electronics", Second Edition, Kluwer Academic Publishers, Dordrecht, The Netherlands, 2001.

[7] Fakham H., Djemaï M. and Busawon K., "Design and practical implementation of a back EMF sliding mode observer for a brushless DC motor", IET Electric Power Applications, 2(6), pp. 353-361, 2008.

[8] Fridman L., Shtessel Y., Edwards C. and Yan X., "Higher-order sliding mode observer for state estimation and input reconstruction in nonlinear systems", International Journal of Robust and Nonlinear Control, 18(4), pp. 399-412, 2008.

[9] Ghanes M., Barbot J. P., "On sliding mode and adaptive observers design for multicell converter", IEEE American Control Conference, St Louis, Missouri, USA, 2009.

[10] Glumineau A., Moog C. and Plestan F., "New algebra-geometric conditions for the linearization by input-output injection", IEEE Transactions on Automatic Control, 41(4), pp. 598-603, 1996.

[11] Hermann R. and Krener A., "Nonlinear controllability and Observability", IEEE Trans. on Automatic Control, 22(9), pp. 728-740, 1977.

[12] Hong Y., "Finite-time stabilization and stabilizability of a class of controllable systems", Systems and Control Letters, 46(4), pp. 231-236, 2002.

[13] Isidori A., "Nonlinear Control Sytems", Springler-Verlag, London, 1999.

[14] Kang W., Barbot J. P. and Xu L., "On the Observability of Nonlinear
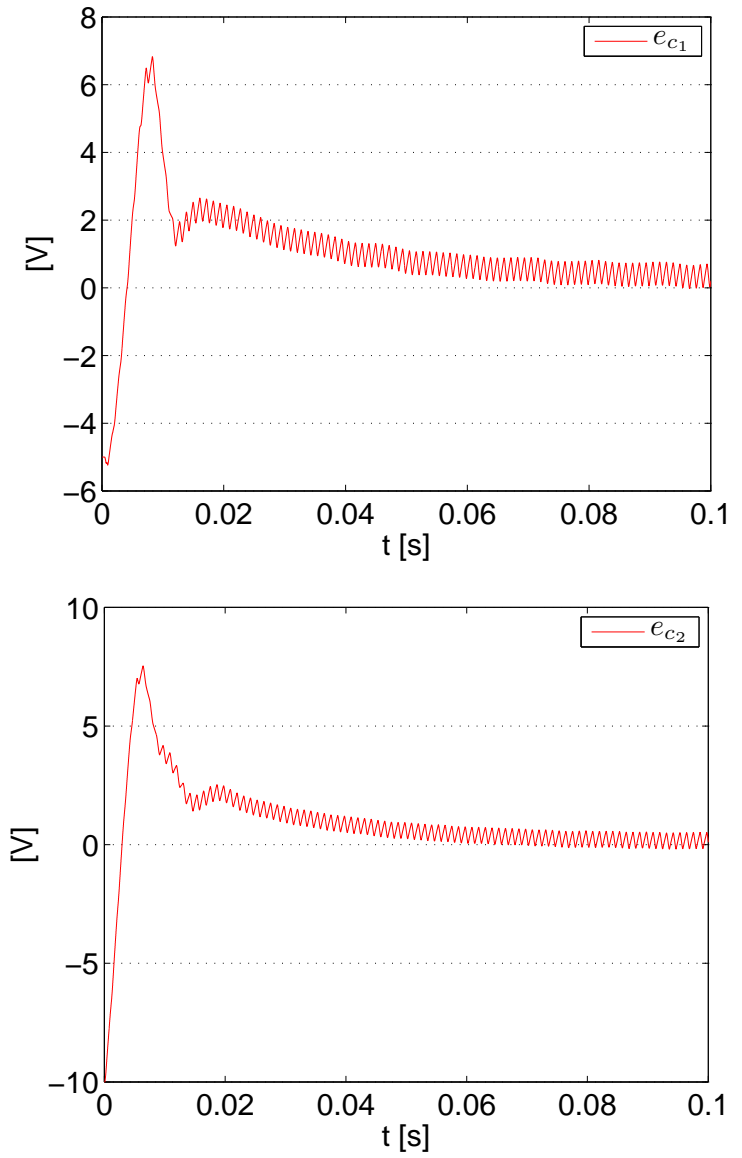

Fig. 6. Observation errors for the finite time observer using $\alpha=\frac{3}{4}$ when $\widehat{R}=1.25 R$

and Switched Systems", Lecture Notes in Control and Information Sciences, Springer Berlin, 2009.

[15] Levant A., "Higher-order sliding modes, differentiation and outputfeedback control”, Int. J. of Control, 76(9/10), pp. 924-941, 2003.

[16] Lygeros J., Johansson K., Simic S., Zhang J. and Sastry S., "Dynamical Properties of Hybrid Automata", IEEE Transations on automatic control, 48(1), pp. 2-14, 2003.

[17] Meynard T. and Foch H., French patent N91,09582 du 25 juillet 1991, dpt international PCT (Europe, Japon, USA, Canada), N92,00652 du 8 juillet 1992, 1991.

[18] Meynard T. A., Foch H., Thomas P., Courault J., Jakob R. and Nahrstaedt M., "Multi-cell converters: basic concepts and industry applications", IEEE Transaction on Industrial Applications, 49(5), pp. 955-964, 2002.

[19] Perruquetti W. and Floquet T., "Homogeneous finite time observer for nonlinear systems with linearizable error dynamics", IEEE Conference on Decision and Control, New-Orleans, USA, 2007.

[20] Perruquetti W., Floquet T. and Moulay E., "Finite time observers: application to secure communication", IEEE Transactions on Automatic Control, 53(1), pp. 356-360, 2008.

[21] Saadaoui H., Manamanni N., Djemaï M., Barbot J. P. and Floquet T., "Exact differentiation and sliding mode observers for switched Lagrangian systems", Nonlinear Analysis : Theory, Methods and Applications, 65(5), pp. 1050-1069, 2006.

[22] Shen Y. and Xia X., "Semi-global finite-time observers for nonlinear systems", Automatica, 44(5), pp. 3152-3156, 2009.

[23] Utkin V., "Sliding modes in control and optimization", SpringerVerlag, Berlin, 1992. 